\title{
Emotional Regulation Difficulties, Personality, and Problematic Smartphone Use
}

\author{
Sharon Horwood and Jeromy Anglim ${ }^{1}$
}

\begin{abstract}
Emotion regulation has been proposed as a mechanism for the development of problematic smartphone use. In addition to examining the relationship between emotional regulation difficulties and problematic smartphone use, the current study sought to be the first to examine the relationship between subscales of emotion regulation difficulties and problematic smartphone use. It also sought to determine whether emotion regulation difficulties provide incremental prediction of problematic smartphone use over and above personality. Participants were 692 Australian university students ( $81 \%$ female; age in years $M=25.23, S D=7.48)$. They completed a measure of problematic smartphone use, the Difficulties in Emotion Regulation Scale, and a measure of Big Five personality (IPIP 120). Overall emotion regulation difficulties $(r=.40)$ and impulse control difficulties $(r=.42)$ were moderately associated with problematic smartphone use, as were the Big Five factors of neuroticism $(r=.43)$ and conscientiousness $(r=-.38)$. Although emotion regulation difficulties predicted problematic smartphone use, they did not provide incremental prediction over and above the Big Five. Findings indicate that personality is a robust predictor of problematic smartphone use. Emotion regulation difficulties, such as impulsivity, offer insights into the specific ways that personality is expressed in problematic smartphone use.
\end{abstract}

Keywords: Emotion regulation; impulsivity; personality; problematic smartphone use; Big Five;

\section{Introduction}

Since their introduction in 2007, smartphones have become an integral part of daily life ${ }^{1}$. Despite the many benefits offered by smartphones, there is an emerging public health concern associated with excessive or problematic use ${ }^{2-4}$. Although there is growing consensus surrounding the health and wellbeing implications of particular

${ }^{1}$ School of Psychology, Deakin University, Geelong, Australia

Please cite as follows (check for updated year, volume, and page numbers):

Horwood, S. \& Anglim, J. (2020). Emotional Regulation Difficulties, Personality, and Problematic Smartphone Use. Cyberpsychology, Behavior, and Social Networking. doi: 10.1089/cyber.2020.0328

Data, analysis scripts, and study materials are available on the OSF at https://osf.io/w3bau

Correspondence concerning this article should be addressed to Sharon Horwood, School of Psychology, Deakin University, Locked Bag 20000, Geelong, 3220 Australia. Email: sharon.horwood@deakin.edu.au 
smartphone usage styles, such as entertainment, communication, or process use ${ }^{5-10}$, the exact mechanisms that explain the development of problematic use remain unclear.

Two proposed dispositional mechanisms are emotion regulation difficulties and personality. A poor ability to regulate emotional responses to negative experiences appears to be associated with greater problematic smartphone use in adults ${ }^{6,11,12}$ and adolescents ${ }^{13-15}$. Similarly, broad personality traits, particularly high neuroticism and low conscientiousness, have also been found to predict greater problematic smartphone use ${ }^{16-23}$. While there is growing evidence that emotion regulation difficulties are associated with problematic smartphone use, there are two major gaps in this program of research. First, no studies have examined which specific aspects of emotion regulation difficulties are most associated with problematic smartphone use. Second, no studies have examined the same association in conjunction with Big Five personality within a single sample. The current research sought to address both of these gaps.

\subsection{Emotion Regulation and Problematic Smartphone Use}

Despite some divergence in definitions ${ }^{24}$, Cole et al. ${ }^{25}$, defines emotion regulation as a predictable pattern of emotion exhibited in response to momentary contextual information. Emotion regulation is particularly concerned with the ability and tendency to regulate emotional responses to negative life events in a socially tolerable way ${ }^{25-27}$. Several meta-analytic reviews e.g. ${ }^{26,28,29-32}$ of the effectiveness of emotion regulation strategies have found that effective cognitive reappraisal correlates with positive health outcomes $(r=.26)$ and negatively with negative health outcomes $(r=-.20)^{31}$, whereas expressive suppression or avoidance correlates positively with negative health outcomes $(r=.34){ }^{30}$. Gratz and Roemer ${ }^{24}$ proposed six separate but related emotion regulation difficulties that contribute to maladaptive emotion regulation: (1) non-acceptance of emotional responses, (2) difficulties engaging in goal directed behavior, (3) impulse control difficulties, (4) lack of emotional awareness, (5) limited access to emotion regulation strategies, and (6) lack of emotional clarity.

Recent research suggests that poor emotion regulation may help to explain various addictive behaviors, including problematic smartphone use ${ }^{9,33,34}$. One theory proposed to explain this effect is the I-PACE model Interaction of Person-AffectCognition-Execution; 35,36 . This model describes various psychological and neurobiological factors that lead to addiction to internet applications. According to this model, people use the internet and relevant devices to regulate their emotion. In particular, a desire to quickly regulate negative emotions can lead to impulsive and habitual use ${ }^{35}$. This impulsive habit can then replace more adaptive strategies and coping skills ${ }^{35,36}$. Furthermore, it seems plausible that people who have emotion regulation difficulties may be more inclined to use their smartphone for such purposes.

To date, only a few studies have examined the role of poor emotion regulation in the development and maintenance of problematic smartphone use. For example, Hoffner and Lee ${ }^{12}$ found that adults often use their smartphones to regulate their emotions, however the study did not investigate how emotion regulation difficulties were associated with problematic smartphone use. Several studies have found that the emotion regulation strategy of expressive suppression is associated with greater problematic smartphone use ${ }^{6,37,38}$. Elhai and Contractor ${ }^{39}$ found that increased use of cognitive reappraisal was indirectly associated with increased problematic use. They 
found that cognitive reappraisal, which can be both an effective and ineffective strategy for regulating emotional responses to negative events, was positively associated with heavier smartphone use, and heavy use of smartphones was associated with increased problematic use. Fu et al ${ }^{13}$ and Extremera, Quintana-Orts [14] found similar results with an adolescent sample whereby emotion regulation difficulties were associated with greater problematic smartphone use. It may be that emotion regulation difficulties are associated with being more distressed and therefore an increase in reliance on smartphones for comfort, reassurance, or emotional distraction in lieu of more adaptive emotion management ${ }^{37}$. Nonetheless, research has not yet considered the combined effect of emotion regulation difficulties and personality on problematic smartphone use.

\subsection{Personality and Problematic Smartphone Use}

Reflecting potentially broader and more distal mechanisms, personality traits predict problematic smartphone use. A growing number of studies have found that of the Big Five, high neuroticism and low conscientiousness predict problematic smartphone use well ${ }^{16,18-20,23,40-43}$. High neuroticism captures a general tendency to experience negative emotions, maladaptive coping strategies, rumination, and addictive tendencies e.g. ${ }^{8,19,20,44,45}$. In contrast, low conscientiousness includes reduced ability to delay gratification and work towards longer term socially-approved goals $^{46}$.

Importantly, individual differences in emotion regulation difficulties are highly correlated with Big Five personality, especially neuroticism e.g. ${ }^{47,48,49}$. For example, Mohammadkani and colleagues ${ }^{48}$ obtained a correlation of -.62 between neuroticism and the difficulties in emotion regulation scale. From a theoretical perspective, neuroticism captures a broad set of individual differences related to the experience of negative emotions which include emotion regulation strategies. It is conceptually feasible that high levels of neuroticism lead to more frequent experiences of negative emotion, for which adaptive emotion regulation strategies are required. If a person who is high in neuroticism also has difficulties in regulating their emotions, the cognitive and affective decision-making heuristics outlined in the I-PACE model may lead to increased use of smartphones as a means of regulating emotions. One of the benefits of examining difficulties in emotion regulation is in the breakdown of subscales to highlight which aspects of emotional experience and regulation are more or less important in predicting behavior such as problematic smartphone use. It is also unclear whether emotion regulation difficulties provide incremental prediction over and above the Big Five factors of personality, or whether they are better understood as a partial expression of neuroticism and potentially other Big Five traits. We note that past research examining the incremental prediction of the 30 facets from the NEO framework in predicting problematic smartphone use found relatively little incremental prediction over and above the Big Five ${ }^{16}$. This may suggest that the broad trait of neuroticism is able to capture the key dispositional individual differences that influence problematic smartphone use. However, it is unclear whether this finding from research on personality facets generalizes to the subscales of emotion regulation difficulties. 


\subsection{The Current Study}

The current study provides a comprehensive examination of the ability of the Big Five factors and emotion regulation difficulties to predict problematic smartphone use in a large adult sample, and address two key gaps in this literature. First, we aimed to assess whether difficulties in emotion regulation provided incremental prediction of problematic smartphone use over and above Big Five personality. While we expected neuroticism to capture much of the effect of emotion regulation difficulties, it was unclear whether emotion regulation difficulties would provide some incremental prediction. Second, we aimed to assess which aspects of emotion regulation difficulties have the strongest associations with problematic smartphone use. In terms of subscales of emotion regulation difficulties, given that impulse control difficulties may be expressed by an inability to regulate smartphone use, we expected this to have a strong association with problematic smartphone use.

\section{Method}

Data, analysis scripts using R, and study materials are available on the OSF at https://osf.io/w3bau

\subsection{Participants and Procedure}

The sample was drawn from an undergraduate psychology unit at an Australian University. Data collection took place across two cohorts from June to December 2018. Prior to completing the surveys, students were asked to indicate whether they consented to their anonymized data being used for research purposes. Data from students who did not consent were excluded. Participants completed an online survey which measured difficulties with emotion regulation, Big Five personality, and problematic smartphone use, along with some additional measures not part of the current study. Several factors helped to ensure that participants were motivated to provide high quality responses. Specifically, students were provided with personalized feedback on their personality that they used in a subsequent assessment task. To remove any sense of obligation, students who chose not to complete the survey they were provided with simulated personality feedback.

The final cleaned sample consisted of 692 participants $(81 \%$ female; age in years $M=25.23, S D=7.48$, range: 18 to 63 ). This was based on an initial sample of 704, of which 12 were removed for one or more of the following reasons: (a) missing data on age or sex $(n=6)$, (b) reporting that they do not use a smartphone $(n=4$; i.e., 99.4\% used a smartphone), (c) providing the same response to all personality items ( $n$ $=1$ ), completing the survey so quickly that they may not have had time to read the items (i.e., less than 17 minutes) $(n=0)$, and $(d)$ reporting an age greater than $90(n=$ $1)$.

\subsection{Materials}

\subsubsection{Big Five Personality}

The Big Five personality factors was measured using the Johnson's 120 item version of the IPIP-NEO ${ }^{50}$. Items were rated on a 5-point scale from $1=$ very inaccurate to $5=$ very accurate. Correlations between corresponding NEO-PI-R and Johnson IPIP NEO 120 Big Five scales have been found to range from .76 and $.87^{50}$. 


\subsubsection{Difficulties in Emotion Regulation}

The Difficulties in Emotion Regulation Scale DERS, ${ }^{24}$ is 36 -item self-report questionnaire designed to assess six aspects of emotion dysregulation: (1) nonacceptance of emotional responses, (2) difficulties engaging in goal-directed behavior, (3) impulse control difficulties, (4) lack of emotional awareness, (5) limited access to emotion regulation strategies, and (6) lack of emotional clarity. Items were rated on a 5-point scale from $1=$ almost never to $5=$ almost always. The six subscales are scored as the mean of constituent items after reversals, where higher scores indicate more difficulties with emotion regulation.

\subsubsection{Problematic Smartphone Use}

Problematic smartphone use was measured using a version of the 21 item Adolescent Preoccupation with Screen Scale ${ }^{51}$ adapted for use with adult samples. The 21 items were scored on a 5-point Likert scale, ranging from 1= 'Strongly disagree' to $5=$ 'Strongly agree'. We adapted the scale for adult use by changing the word 'parents' to 'family and friends'. The word 'screens' was replaced with 'smartphone'.

\section{Results}

Table 1 presents descriptive statistics, Cronbach's alpha reliability, and scale intercorrelations. Mean problematic smartphone use was $2.55(S D=0.70)$. An examination of the correlations between personality and emotion regulation difficulties highlights the potential for, and limits of, incremental prediction. First, neuroticism correlated very highly with overall emotion regulation difficulties, $r=.72$. Second, most emotion regulation difficulty subscales correlated highly with each other and with neuroticism. Emotion regulation difficulty subscales tended to have moderate negative correlations with both extraversion and conscientiousness. That said, impulse control had a stronger negative correlation with conscientiousness, and lack of emotional awareness correlated less with neuroticism and had a relatively larger negative correlation with openness. Third, age and sex had only small to moderate correlations with personality and emotional regulation. Interestingly the tendency for females to report higher levels of neuroticism than males was less pronounced in relation to difficulties with emotion regulation.

Both personality and emotion regulation difficulties correlated with problematic smartphone use. In terms of personality, problematic smartphone use had a strong positive correlation with neuroticism and a strong negative correlation with conscientiousness, consistent with past research. Overall emotion regulation difficulties scale, and most of the subscales, were also positively correlated with problematic smartphone use. Correlations were strongest for impulse control difficulties, limited access to emotion regulation strategies, and overall emotion regulation difficulties. Interestingly, the correlation with lack of emotional awareness was close to zero.

Table 2 presents coefficients and variance explained from regression models predicting problematic smartphone use from various predictor sets: (1) neuroticism, (2) the Big Five, (3) overall emotion regulation difficulties, (4) the six subscales of emotion regulation difficulties, (5) Big Five and overall emotion regulation difficulties, and (6) the Big Five and the six subscales of emotion regulation difficulties. Comparisons between these models allows for assessment of the 
incremental prediction of personality and emotion regulation difficulties. Overall, neuroticism was the best single predictor of problematic smartphones use, followed by overall emotion regulation difficulties. In general, the Big Five predicted problematic smartphone use substantially better than just neuroticism. Interestingly, in addition to lower levels of conscientiousness being important, these models also suggested that after controlling for the other factors of the Big Five, greater extraversion predicted more problematic use, which contrasts with the bivariate correlations. Models with the six emotion regulation difficulties subscales performed only slightly better at predicting problematic smartphone use than a model with just the overall scale. This model highlighted impulse control as the most important predictor. Interestingly, the coefficient for lack of emotional awareness was the opposite sign to the other predictors. Overall, the addition of either overall emotion regulation difficulties or the subscales did not improve prediction over and above the Big Five.

\section{Discussion}

The current study examined the relationship between difficulties in emotion regulation and problematic smartphone use. Several important findings emerged. First, high neuroticism and low conscientiousness again emerged as the key personality traits predicting problematic smartphone use. Second, emotion regulation difficulties had a similarly strong, albeit slightly smaller, correlation with problematic smartphone use than did neuroticism. Third, impulse control and limited access to emotion regulation strategies were the emotion regulation difficulties subscales with the strongest correlations with problematic smartphone use. Fourth, emotion regulation difficulties did not provide meaningful incremental prediction over and above neuroticism or the Big Five. Overall, the findings have implications for how the dispositional mechanisms of personality and emotional regulation are conceptualized in relation to problematic smartphone use.

\subsection{Emotion Regulation Difficulties and Problematic Smartphone Use}

The results were consistent with past research that has reported moderate to strong positive relationship between poor emotion regulation and problematic smartphone use ${ }^{6,11-14,37,38}$. Although, the relationship between overall emotion regulation difficulties and problematic smartphone use is interesting, the subscale associations indicate a clearer picture of the specific aspects of emotion regulation difficulties that are important to understanding problematic smartphone use. Impulse control difficulties and limited access to emotion regulation strategies had the strongest associations with problematic usage, and their roles seem to have clear face validity. Impulse control difficulties refer to a feeling of loss of control, or actual loss of control, over one's behavior when experiencing negative emotion ${ }^{24}$. When experiencing negative emotion, people who have difficulty controlling behavioral impulses typically fail to keep their reactions to the feeling in check. Reacting impulsively to try to alleviate negative emotions could mean using a smartphone for cognitive distraction and emotional suppression, common emotion-focused coping strategies 6, 12, 37 . Similarly, people who report limited access to emotion regulation strategies tend to a believe that little or nothing can be done to improve a negative situation ${ }^{24}$ and may also rely on emotion-focused coping strategies via their smartphone.

Although difficulties with emotion regulation appears to be important in understanding problematic smartphone use, it did not provide incremental prediction 
over and above the Big Five. In fact, neuroticism alone was a slightly better predictor than overall emotion regulation difficulties. The correlations between emotion regulation difficulties, including the subscales, and the Big Five offer a meaningful explanation for this effect. Neuroticism correlates very strongly with overall emotion regulation difficulties and most of the subscales. It is important to note, however, that impulsive behavioral decisions that are aimed at regulating negative emotional experiences can take the place of effective coping strategies ${ }^{35,36}$. After overall emotion regulation difficulties, the subscales of limited access to emotion regulation strategies and impulse control difficulties are the two highest correlations with neuroticism, and these are consistent with the general processes outline in the I-PACE model ${ }^{35,36}$. The central characteristics of neuroticism are negative emotions such as worry, fear, and anxiety, and, importantly, impulsivity ${ }^{52}$. Given the very strong relationship between neuroticism and emotion regulation difficulties, a plausible explanation would be that emotion regulation difficulties are largely an expression of the broader and more distal trait of neuroticism.

Importantly, the Big Five predicted problematic smartphone use better than neuroticism alone. This reflects the importance of low conscientiousness in problematic smartphone use. Conscientiousness correlated much less with emotion regulation difficulties, although the overlap was most notable in relation to impulse control difficulties and limited access to emotion regulation strategies. Conscientiousness is characterized by self-regulation, pursuing socially approved goals, and an ability to delay short-term gratification for longer-term goals. Much of the broad and important role of conscientiousness in protecting against problematic smartphone use is not captured by the subscales of emotion regulation difficulties.

\subsection{Limitations and Future Research}

The current study had several limitations that should be noted. First, the research is cross-sectional, which limits any ability to make causal inferences. Future research could examine the real-time processes of emotion regulation and smartphone use using experience sampling and related technologies. Second, it is unclear whether the relationships between self-reported measures of personality, emotion regulation difficulties and problematic smartphone are prone to common method bias. Future research could test the usefulness of self-report data and the effect of neurotic tendencies by including objective smartphone usage data or other-ratings of personality and problematic smartphone use in analyses.

\subsection{Conclusion}

The present study aimed to address two gaps in the emotion regulationproblematic smartphone use literature. The results indicate that the aspects of emotion regulation difficulties that are most associated with problematic smartphone use are impulse control difficulties, and lack of access to effective emotion regulation strategies. The results also indicated that while broad personality traits predict problematic smartphone use well, emotion regulation is a useful framework for contextualizing the influence of broad traits. By understanding the mechanisms by which personality broadly, and emotion regulation specifically, influence thoughts and behaviors associated with problematic smartphone use, practical therapies or behavior change interventions can be developed. 


\section{References}

1. Taylor, K. and L. Silver, Smartphone Ownership Is Growing Rapidly Around the World, but Not Always Equally. 2019, Pew Research Center

2. $\quad$ Billieux, J., Problematic use of the mobile phone: a literature review and a pathways model. 2012. 8(4): p. 299-307.

3. Elhai, J.D., et al., Problematic smartphone use: A conceptual overview and systematic review of relations with anxiety and depression psychopathology. Journal of affective disorders, 2017. 207: p. 251-259.

4. van Velthoven, M.H., J. Powell, and G. Powell, Problematic smartphone use: digital approaches to an emerging public health problem. 2018, SAGE Publications Sage UK: London, England.

5. $\quad$ Elhai, J.D., et al., Types of smartphone usage and relations with problematic smartphone behaviors: The role of content consumption vs. social smartphone use. Cyberpsychology: Journal of Psychosocial Research on Cyberspace, 2017. 11(2).

6. Elhai, J.D., et al., Fear of missing out, need for touch, anxiety and depression are related to problematic smartphone use. Computers in Human Behavior, 2016. 63: p. 509-516.

7. $\quad$ Elhai, J.D., et al., Non-social features of smartphone use are most related to depression, anxiety and problematic smartphone use. Computers in Human Behavior, 2017. 69: p. 75-82.

8. Anglim, J., et al., Predicting psychological and subjective well-being from personality: A meta-analysis. Psychological Bulletin, 2020. 146(4): p. 279.

9. Rozgonjuk, D. and J.D. Elhai, Emotion regulation in relation to smartphone use: Process smartphone use mediates the association between expressive suppression and problematic smartphone use. Current Psychology, 2019.

10. Rozgonjuk, D., et al., Non-social smartphone use mediates the relationship between intolerance of uncertainty and problematic smartphone use: Evidence from a repeated-measures study. Computers in Human Behavior, 2019. 96: p. $56-62$.

11. Yildiz, M.A., Emotion regulation strategies as predictors of internet addiction and smartphone addiction in adolescents. Journal of Educational Sciences and Psychology, 2017. 7(1).

12. Hoffner, C. and S. Lee, Mobile Phone Use, Emotion Regulation, and Well-Being. Cyberpsychology, Behavior, and Social Networking, 2015. 18(7): p. 411-416.

13. Fu, L., et al., Can emotion regulation difficulty lead to adolescent problematic smartphone use? A moderated mediation model of depression and perceived social support. Children and Youth Services Review, 2020. 108.

14. Extremera, N., et al., The role of cognitive emotion regulation strategies on problematic smartphone use: Comparison between problematic and non-problematic adolescent users. International Journal of Environmental Research and Public Health, 2019. 16(17).

15. Gül, H., et al., Cyberbullying among a clinical adolescent sample in Turkey: effects of problematic smartphone use, psychiatric symptoms, and emotion regulation difficulties. Psychiatry and Clinical Psychopharmacology, 2019. 29(4): p. 547-557.

16. Horwood, S. and J. Anglim, Personality and problematic smartphone use: A facet-level analysis using the Five Factor Model and HEXACO frameworks. Computers in Human Behavior, 2018.

17. Carvalho, L.F., C.P. Sette, and B.L. Ferrari, Problematic smartphone use relationship with pathological personality traits: Systematic review and meta-analysis. Cyberpsychology, 2018. 12(3).

18. Blackwell, D., et al., Extraversion, neuroticism, attachment style and fear of missing out as predictors of social media use and addiction. 2017. 116: p. 69-72.

19. Gao, T., et al., Neuroticism and quality of life: Multiple mediating effects of smartphone addiction and depression. Psychiatry Research, 2017. 258: p. 457-461.

20. Hussain, Z., M.D. Griffiths, and D. Sheffield, An investigation into problematic smartphone use: The role of narcissism, anxiety, and personality factors. Journal of Behavioral Addictions, 2017. 6(3): p. 378-386.

21. Kim, Y., et al., Personality factors predicting smartphone addiction predisposition: Behavioral Inhibition and Activation Systems, Impulsivity, and Self-Control. PLoS ONE, 2016. 11(8).

22. Takao, M., Problematic mobile phone use and big-five personality domains. Indian Journal of Community Medicine, 2014. 39(2): p. 111-113.

23. Roberts, J.A., et al., I need my smartphone: A hierarchical model of personality and cell-phone addiction. 2015. 79: $\mathrm{p}$. 13-19.

24. Gratz, K.L. and L. Roemer, Multidimensional assessment of emotion regulation and dysregulation: Development, factor structure, and initial validation of the difficulties in emotion regulation scale. Journal of psychopathology and behavioral assessment, 2004. 26(1): p. 41-54.

25. Cole, P.M., M.K. Michel, and L.O.D. Teti, The development of emotion regulation and dysregulation: A clinical perspective. Monographs of the society for research in child development, 1994. 59(2 - 3): p. 73-102.

26. Gross, J.J., Emotion regulation: Affective, cognitive, and social consequences. 2002. 39(3): p. 281-291.

27. Estévez, A., et al., Attachment and emotion regulation in substance addictions and behavioral addictions. Journal of Behavioral Addictions, 2017. 6(4): p. 534-544.

28. Gross, J.J. and H. Jazaieri, Emotion, emotion regulation, and psychopathology: An affective science perspective. Clinical Psychological Science, 2014. 2(4): p. 387-401.

29. Chesney, S.A., S.C. Timmer-Murillo, and N.S. Gordon, Establishment and replication of emotion regulation profiles: implications for psychological health. Anxiety, Stress \& Coping, 2019. 32(3): p. 329-345. 
30. Aldao, A., S. Nolen-Hoeksema, and S. Schweizer, Emotion-regulation strategies across psychopathology: A metaanalytic review. Clinical psychology review, 2010.30(2): p. 217-237.

31. Hu, T., et al., Relation between Emotion Regulation and Mental Health: A Meta-Analysis Review. Psychological Reports, 2014. 114(2): p. 341-362.

32. Webb, T.L., E. Miles, and P. Sheeran, Dealing with feeling: A meta-analysis of the effectiveness of strategies derived from the process model of emotion regulation. Psychological Bulletin, 2012. 138(4): p. 775-808.

33. Van Deursen, A., et al., Modeling habitual and addictive smartphone behavior: The role of smartphone usage types, emotional intelligence, social stress, self-regulation, age, and gender. Computers in human behavior, 2015. 45: p. 411420.

34. Loid, K., K. Täht, and D. Rozgonjuk, Do pop-up notifications regarding smartphone use decrease screen time, phone checking behavior, and self-reported problematic smartphone use? Evidence from a two-month experimental study. Computers in Human Behavior, 2020. 102: p. 22-30.

35. Brand, M., et al., The Interaction of Person-Affect-Cognition-Execution (I-PACE) model for addictive behaviors: Update, generalization to addictive behaviors beyond internet-use disorders, and specification of the process character of addictive behaviors. Neuroscience \& Biobehavioral Reviews, 2019. 104: p. 1-10.

36. Brand, M., et al., Integrating psychological and neurobiological considerations regarding the development and maintenance of specific Internet-use disorders: An Interaction of Person-Affect-Cognition-Execution (I-PACE) model. Neuroscience \& Biobehavioral Reviews, 2016. 71: p. 252-266.

37. Rozgonjuk, D. and J. Elhai, Emotion regulation in relation to smartphone use: Process smartphone use mediates the association between expressive suppression and problematic smartphone use. Current Psychology, 2019.

38. Elhai, J., et al., Depression and emotion regulation predict objective smartphone use measured over one week. Personality and Individual Differences, 2018. 133: p. 21-28.

39. Elhai, J. and A.A. Contractor, Examining latent classes of smartphone users: Relations with psychopathology and problematic smartphone use. Computers in Human Behavior, 2018. 82: p. 159-166.

40. Ehrenberg, A., et al., Personality and self-esteem as predictors of young people's technology use. 2008. 11(6): p. 739741.

41. Demirhan, E., C. Randler, and M.B.J.C.i. Horzum, Is problematic mobile phone use explained by chronotype and personality? 2016. 33(7): p. 821-831.

42. de Carvalho Leite, J.C., et al., Social support needs for equity in health and social care: a thematic analysis of experiences of people with chronic fatigue syndrome/myalgic encephalomyelitis. International Journal for Equity in Health, 2011. 10: p. 46-46.

43. Toda, M., et al., Relationship of smartphone dependence to general health status and personality traits among university students. Open Journal of Preventive Medicine, 2016. 6(10): p. 215.

44. $\quad$ Elhai, J., et al., Rumination and negative smartphone use expectancies are associated with greater levels of problematic smartphone use: A latent class analysis. Psychiatry Research, 2020. 285.

45. Squires, L.R., et al., Psychological Distress, Emotion Dysregulation, and Coping Behaviour: a Theoretical Perspective of Problematic Smartphone Use. International Journal of Mental Health and Addiction, 2020.

46. Bogg, T. and B.W. Roberts, Conscientiousness and health-related behaviors: a meta-analysis of the leading behavioral contributors to mortality. Psychological bulletin, 2004. 130(6): p. 887.

47. John, O.P. and J.J. Gross, Healthy and unhealthy emotion regulation: Personality processes, individual differences, and life span development. Journal of personality, 2004. 72(6): p. 1301-1334.

48. Mohammadkhani, P., et al., The Role of Neuroticism and Experiential Avoidance in Predicting Anxiety and Depression Symptoms: Mediating Effect of Emotion Regulation. Iranian journal of psychiatry and behavioral sciences, 2016. 10(3): p. e5047-e5047.

49. Wang, L., Z. Shi, and H. Li, Neuroticism, extraversion, emotion regulation, negative affect and positive affect: The mediating roles of reappraisal and suppression. Social Behavior and Personality: an international journal, 2009. 37(2): p. 193-194.

50. Johnson, J.A., Measuring thirty facets of the Five Factor Model with a 120-item public domain inventory: Development of the IPIP-NEO-120. Journal of Research in Personality, 2014. 51: p. 78-89.

51. Hunter, S.C., et al., Development of the Adolescent Preoccupation with Screens Scale. BMC public health, 2017. 17(1): p. 652.

52. Barlow, D.H., et al., The nature, diagnosis, and treatment of neuroticism: Back to the future. 2014. 2(3): p. 344-365. 


\section{Table 1}

Correlation matrix of Problematic Smartphone Usage, Emotion Regulation Difficulties, Personality, and Demographics

\begin{tabular}{|c|c|c|c|c|c|c|c|c|c|c|c|c|c|c|c|}
\hline Variable & 1 & 2 & 3 & 4 & 5 & 6 & 7 & 8 & 9 & 10 & 11 & 12 & 13 & 14 & 15 \\
\hline $\begin{array}{l}\text { 1. Problematic smartphone } \\
\text { usage }\end{array}$ & & & & & & & & & & & & & & & \\
\hline $\begin{array}{l}\text { 2. Overall emotion regulation } \\
\text { difficulties }\end{array}$ & .40 & & & & & & & & & & & & & & \\
\hline $\begin{array}{l}\text { 3. Nonacceptance of } \\
\text { emotional responses }\end{array}$ & .31 & .82 & & & & & & & & & & & & & \\
\hline $\begin{array}{l}\text { 4. Difficulties engaging in } \\
\text { goal-directed behavior }\end{array}$ & .31 & 68 & .45 & & & & & & & & & & & & \\
\hline 5. Impulse control difficulties & .42 & .81 & .57 & .56 & & & & & & & & & & & \\
\hline $\begin{array}{l}\text { 6. Lack of emotional } \\
\text { awareness }\end{array}$ & .09 & .54 & .39 & .11 & .23 & & & & & & & & & & \\
\hline $\begin{array}{l}\text { 7. Limited access to emotion } \\
\text { regulation strategies }\end{array}$ & .38 & .90 & .67 & .61 & .75 & .35 & & & & & & & & & \\
\hline 8. Lack of emotional clarity & .26 & .72 & .54 & .33 & .43 & .58 & .53 & & & & & & & & \\
\hline 9. Neuroticism & .43 & .72 & .56 & .54 & .63 & .29 & .69 & .47 & & & & & & & \\
\hline 10. Extraversion & -.05 & -.38 & -.30 & -.22 & -.19 & -.35 & -.37 & -.32 & -.49 & & & & & & \\
\hline 11. Openness & -.03 & -.04 & -.01 & .04 & .07 & -.29 & .01 & -.07 & -.02 & .17 & & & & & \\
\hline 12. Agreeableness & -.18 & -.19 & -.12 & -.02 & -.23 & -.20 & -.16 & -.15 & -.11 & .06 & .21 & & & & \\
\hline 13. Conscientiousness & -.38 & -.51 & -.34 & -.38 & -.51 & -.22 & -.45 & -.38 & -.49 & .18 & -.08 & .29 & & & \\
\hline 14. Female & .15 & .09 & .08 & .12 & .13 & -.06 & .08 & .03 & .21 & .05 & -.01 & .18 & .03 & & \\
\hline 15. Age & -.19 & -.18 & -.13 & -.08 & -.14 & -.10 & -.18 & -.18 & -.21 & -.04 & .15 & .15 & .17 & -.04 & \\
\hline Alpha reliability & .92 & .95 & .91 & .89 & .90 & .83 & .92 & .87 & .91 & .89 & .84 & .86 & .90 & & \\
\hline$M$ & 2.55 & 2.58 & 2.76 & 3.46 & 2.38 & 2.09 & 2.58 & 2.34 & 3.01 & 3.27 & 3.52 & 3.93 & 3.65 & 0.81 & 25.23 \\
\hline$S D$ & 0.70 & 0.65 & 0.98 & 0.85 & 0.90 & 0.64 & 0.89 & 0.83 & 0.67 & 0.57 & 0.49 & 0.47 & 0.55 & 0.39 & 7.48 \\
\hline
\end{tabular}

Note. Absolute correlations greater than or equal to $.08, .10$, and .13 are significant at $.05, .01$, and .001 respectively. Absolute correlations greater than or equal to .20 are bolded. Female is coded $0=$ Male, $1=$ Female. 


\section{Table 2}

Standardized Betas and Variance Explained for Regression Models Predicting Problematic Smartphone Use

\begin{tabular}{|c|c|c|c|c|c|c|}
\hline Predictor & M1 & M2 & M3 & M4 & M5 & M6 \\
\hline Neuroticism & $.43^{* * *}$ & $.43 * * *$ & & & $.34^{* * *}$ & $.30^{* * *}$ \\
\hline Extraversion & & $.21 * * *$ & & & $.22 * * *$ & $.18^{* * *}$ \\
\hline Openness & & -.06 & & & -.06 & $-.09^{*}$ \\
\hline Agreeableness & & $-.08 *$ & & & -.07 & -.07 \\
\hline Conscientiousness & & $-.19 * * *$ & & & $-.16^{* * *}$ & $-.16^{* * *}$ \\
\hline Overall emotion regulation difficulties & & & $.40 * * *$ & & $.14^{* *}$ & \\
\hline Nonacceptance of emotional responses & & & & .05 & & .04 \\
\hline Difficulties engaging in goal-directed behavior & & & & .07 & & .02 \\
\hline Impulse control difficulties & & & & $.28 * * *$ & & .10 \\
\hline Lack of emotional awareness & & & & -.08 & & $-.10^{*}$ \\
\hline Limited access to emotion regulation strategies & & & & .07 & & .04 \\
\hline Lack of emotional clarity & & & & $.10^{*}$ & & .06 \\
\hline Adjusted $R^{2}$ & $.18^{* * *}$ & $.26 * * *$ & $.16^{* * *}$ & $.19 * * *$ & $.26 * * *$ & $.27^{* * *}$ \\
\hline
\end{tabular}

